\title{
Spinal manipulation and mobilisation for back and neck pain: a blinded review
}

\author{
B W Koes, W J J Assendelft, G J M G van der Heijden, L M Bouter, P G Knipschild
}

\begin{abstract}
Objective-To assess the efficacy of spinal manipulation for patients with back or neck pain.

Design-Computer aided search for published papers and blinded assessment of the methods of the studies.

Subjects -35 randomised clinical trials comparing spinal manipulation with other treatments.

Main outcome measures-Score for quality of methods (based on four main categories: study population, interventions, measurement of effect, and data presentation and analysis) and main conclusion of author(s) with regard to spinal manipulation.
\end{abstract}

Results-No trial scored 60 or more points (maximum score 100) suggesting that most were of poor quality. Eighteen studies $(51 \%)$ showed favourable results for manipulation. In addition, five studies $(14 \%)$ reported positive results in one or more subgroups. Of the four studies with 50-60 points, one reported that manipulation was better, two reported that manipulation was better in only a subgroup, and one reported that manipulation was no better or worse than reference treatment. Eight trials attempted to compare manipulation with some placebo, with inconsistent results.

Conclusions-Although some results are promising, the efficacy of manipulation has not been convincingly shown. Further trials are needed, but much more attention should be paid to the methods of study.

\section{Introduction}

About $80 \%$ of people in Western countries will Fortunately, the disease is usually self limiting. Most patients recover from an attack of back pain within six weeks, irrespective of the type of treatment given, although the recurrence rate is high. ${ }^{23}$ Despite the common occurrence of back pain its management remains controversial. A wide variety of therapeutic possibilities exists, but no single treatment seems to be superior to others. ${ }^{4}$ Spinal manipulation or mobilisation are widely used for treating back pain, and their efficacy has been studied in randomised clinical trials. ${ }^{5}$ The similarities and differences between the several manipulative techniques available are not always clear. However, there seems to be agreement that manipulation involves a high velocity thrust to a joint beyond its restricted range of movement. Mobilisation uses low velocity passive movements within or at the limit of joint range. ${ }^{6}$ Throughout this article we will use manipulation to cover both manipulation and mobilisation.

The rationale given for manipulation in the management of back and neck pain ranges from reduction of a bulging disk, correction of the internal displacement of disc fragments, and freeing of adhesions around a prolapsed disc or facet joints to inhibition of transmission of nociceptive impulses. ${ }^{78}$ Whether manipulation trials, but the outcome of such trials may be biased by flaws in the methods of the study. We present a critical experience back pain at some time during their lives. ${ }^{12}$ is effective can be evaluated only in randomised clinical

review of the available randomised clinical trials of spinal manipulation for back and neck pain. Strong emphasis will be put on the methods of the studies included.

\section{Methods}

A MEDLINE literature search was carried out for the period 1966-90 (keywords: backache, musculoskeletal diseases, joint diseases, manipulation, osteopathy, chiropractic, evaluation studies, outcome and process assessment). In addition, the references given in relevant publications were further examined. Abstracts and unpublished studies were not selected. Studies had to meet the following criteria: the (experimental) treatment regimen included manipulation of the spine (additional interventions were allowed); the subjects had back or neck pain; the study was a randomised clinical trial.

All trials were scored according to the criteria listed in table I. The criteria are based on generally accepted principles of intervention research. ${ }^{10}$ The criteria were developed by Ter Riet et $a l^{11}$ and have been modified for this study. Each criterion is given a weigh and the maximum score was set at 100 points for each study. All publications were blinded for author(s), journal, and outcome by one of us (BWK). Subsequently, the methodological quality of the studies was assessed by two blinded reviewers (WJJA, GJMGH) independently. In a subsequent meeting they (stil blinded) tried to reach consensus on each criterion they disagreed about. When disagreement persisted a third blinded reviewer (LMB) made the decision. The assessments resulted in a hierarchical list in which higher scores indicate studies with better methods. The outcome of the studies will be discussed in relation to their method scores.

A study was determined to be positive if the author concluded (in their abstract or conclusions, or both) that manipulation was more effective than the refer-

TABLE I-Criteria for assessing methods of randomised clinical trials of manipulation for back and neck pain

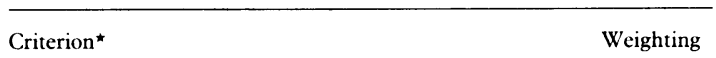

\section{Study population}

A Comparability of relevant baseline characteristics

B Comparability of relevant baseline

D Drop outs described for each study group separately

$\mathrm{E}<20 \%$ Loss to follow up

$<20 \%$ Loss to follow up
$<10 \%$ Loss to follow up

$\mathrm{F}>50$ Subjects in the smallest group

$>100$ Subjects in the smallest group

Interventions

$\mathrm{G}$ Interventions included in protocol and described

H Pragmatic study

I Cointerventions avoided

$\mathrm{J}$ Placebo controlled

$\mathrm{K}$ Mentioning good qualification of manipulative therapist

Effect

L Patients blinded

M Outcome measures relevant

$\mathrm{N}$ Blinded outcome assessments

O Follow up period adequate

Data presentation and analysis

$P$ Intention to treat analysis

$Q$ Frequencies of most important outcomes presented for each treatment group

Mr Koes.

Further details given in appendix. 
ence treatment. Sometimes the authors reported favourable outcomes for manipulation in only a subgroup of the study population. In a negative study the authors reported no differences between the study treatments or better results with the reference treatment. Short term outcome refers to effect measurements during or just after the intervention period. Long term outcome refers to outcome measurements at least three months aiter randomisation.

\section{Results}

Thirty eight trials met the inclusion criteria. Three trials were found to be reported twice and the publication with the lowest method score in each case was excluded..$^{72}{ }^{13}$ Table II gives the remaining 35 trials in a hierarchical order according to their methodological score. Thirty trials were in patients with back pain and five in those with neck pain. Initially the two blinded reviewers disagreed over the criterion in $276(20 \%)$ of the 1400 instances. Usually this seemed to be due to errors in reading. After their consensus meeting disagreement was reduced to four instances, for which the third blinded reviewer made the final decision.

No trial scored 60 or more points, and only four studies (three of back pain, one of neck pain) scored more than 50 points, suggesting a general poor quality. The most prevalent methodological problems were the proper description of drop outs, the small size of the study population, the lack of a placebo group, the blinding of patients, and the blinded measurements of effect. In 18 trials $(51 \%)$ the authors reported better results for manipulation than the reference treatment (for example, short wave diathermy, massage, exercises, analgesics, or placebo treatment). In addition, five other trials reported better results in only a subgroup of the study population. In 11 studies manipulation seemed to be no more effective than the reference treatment

Considering the four trials with methodological scores of 50-60 points, one reported positive results and one negative results, and two studies reported better results in only a subgroup of the study population. Considering the nine trials with method scores of $\stackrel{\vec{D}}{\rightarrow}$ 40-50 points two reported positive results, six negative, $\overline{0}$ and one better results in only a subgroup. Only 14 흠 studies measured the effect at least three months after $\frac{\bar{\rho}}{\vec{D}}$ randomisation, only four of which reported long term $\mathbb{\perp}$ positive effects of manipulation.

The figure presents the relation between the method ${ }^{\infty}$ score and the outcome of the study. Included are the 18. positive studies and the 11 negative studies. The five trials reporting positive results of manipulation in only $\stackrel{\sigma}{\omega}$ a subgroup were omitted because the labelling as $\frac{0}{3}$ positive or negative would be ambiguous for these. One study in which the author did not draw a conclusion was also omitted. Only three of the $18 \%$ positive studies $(17 \%)$ scored 40 points or more. In $\underset{+}{\infty}$ contrast, seven of the 11 negative studies $(64 \%)$ scored 40 points or more. In general the negative studies $\vec{N}$ seemed to have higher method scores.

Table III presents the comparison of manipulation with other conservative treatments and gives the main $N$ characteristics of the forms of manipulation included $\omega$ in the trials. Manipulation was given alone or in combination with other treatments. The reference

TABLE II -Randomised trials on the efficacy of manipulation for back pain and neck pain in order of methods score

\begin{tabular}{|c|c|c|c|c|c|c|c|c|c|c|c|c|c|c|c|c|c|c|c|c|}
\hline \multirow[b]{2}{*}{ Study } & \multicolumn{17}{|c|}{ Methods criteria } & \multirow{2}{*}{$\begin{array}{c}\text { Total } \\
\text { score } \\
100\end{array}$} & \multirow{2}{*}{ Indication $^{\star}$} & \multirow{2}{*}{ Conclusion $t$} \\
\hline & $\begin{array}{l}\text { A } \\
2\end{array}$ & $\begin{array}{l}\mathrm{B} \\
5\end{array}$ & $\begin{array}{l}\mathrm{C} \\
4\end{array}$ & $\begin{array}{l}\mathrm{D} \\
3\end{array}$ & $\begin{array}{l}\mathrm{E} \\
4\end{array}$ & $\begin{array}{c}\mathrm{F} \\
12\end{array}$ & $\begin{array}{l}\mathrm{G} \\
10\end{array}$ & $\begin{array}{c}\mathrm{H} \\
5\end{array}$ & $\begin{array}{l}\text { I } \\
5\end{array}$ & $\begin{array}{l}J \\
5\end{array}$ & $\begin{array}{l}\mathrm{K} \\
5\end{array}$ & $\begin{array}{l}\mathrm{L} \\
5\end{array}$ & $\begin{array}{l}M \\
10\end{array}$ & $\begin{array}{l}\mathrm{N} \\
10\end{array}$ & $\begin{array}{l}\mathrm{O} \\
5\end{array}$ & $\begin{array}{l}P \\
5\end{array}$ & $\begin{array}{l}Q \\
5\end{array}$ & & & \\
\hline \multicolumn{21}{|l|}{ Back pain trials: } \\
\hline MacDonald, Bell ${ }^{14}$ & 1 & 4 & & 3 & 4 & & 10 & 5 & 5 & & 5 & & 6 & & 3 & 5 & 5 & 56 & Acute plus chronic low back pain & $\begin{array}{l}\text { Positive (only in patients with pain of } 2-4 \text { week } \\
\text { duration not those with }<2 \text { weeks or }>4 \text { wee }\end{array}$ \\
\hline Hadler $e t a l^{15}$ & 1 & 3 & & & 4 & & 10 & 5 & 5 & & 5 & 3 & 4 & & 3 & 5 & 5 & 53 & Acute low back pain & $\begin{array}{l}\text { Positive (only in patients with pain of } 2-4 \text { week } \\
\text { duration not those with }<2 \text { weeks) }\end{array}$ \\
\hline $\begin{array}{l}\text { Ongley et al } \text { l' }^{1 \hbar} \\
\text { Bergquist-Ullman, }\end{array}$ & 2 & 4 & 2 & & 4 & & 5 & 5 & & & & 5 & 4 & 4 & 5 & 5 & 5 & 50 & Chronic low back pain & Positive \\
\hline Larsson & 2 & 1 & 2 & & 4 & 6 & 10 & 5 & & 5 & 5 & 2 & 2 & & 5 & & & 49 & Acute low back pain & $\begin{array}{l}\text { Positive compared with placebo } \\
\text { Negative compared with back school }\end{array}$ \\
\hline Meade et al ${ }^{18}$ & & 4 & 4 & & 2 & 12 & & 5 & & & 5 & & 6 & & 5 & & 5 & 48 & Acute plus chronic low back pain & Positive \\
\hline Gibson $e t a l^{19}$ & 2 & 3 & & 3 & 4 & & & 5 & 5 & 5 & 5 & & 4 & 2 & 3 & & 5 & 46 & (Sub)acute low back pain & Negative \\
\hline Sims-Williams et al ${ }^{20}$ & 1 & 2 & & & 4 & & 5 & 5 & 5 & & 5 & & 6 & 2 & 5 & & 5 & 45 & Acute plus chronic low back pain & Negative \\
\hline Helliwell, Cunliffe ${ }^{21}$ & 1 & 3 & & 3 & 4 & & 5 & 5 & 5 & & & & 2 & 2 & 3 & 5 & 5 & 43 & Acute low back pain & Negative \\
\hline Doran, Newell ${ }^{22}$ & 1 & & & & 2 & 12 & & 5 & & & 5 & 3 & 4 & 2 & 3 & & 5 & 42 & Acute plus chronic low back pain & Negative \\
\hline Mathews et al ${ }^{3}$ & & 2 & 0 & 3 & 2 & 12 & 5 & 5 & & & & & 2 & & 5 & & 5 & 41 & Acute back pain & $\begin{array}{l}\text { Positive (only in patients in whom straight leg } \\
\text { raising was limited) }\end{array}$ \\
\hline Evans $e t a l^{24}$ & & & 2 & & 2 & & 5 & 5 & 5 & & 5 & & 6 & 2 & 3 & & 5 & 40 & Chronic low back pain & Positive \\
\hline Glover $e t a l^{5}$ & & 3 & 4 & 3 & 4 & & 5 & & 5 & 5 & & & 2 & & 3 & 5 & & 39 & Acute back pain & Negative \\
\hline Coxhead et al ${ }^{2 \mathrm{~h}}$ & 1 & 1 & & & 2 & 12 & & 5 & & & & & 4 & & 3 & 5 & 5 & 38 & Sciatic symptoms & Positive \\
\hline Waagen et al ${ }^{2}$ & 1 & 2 & & & & & 5 & 5 & 5 & & 5 & 5 & 4 & 2 & 3 & & & 37 & Chronic low back pain & Positive \\
\hline Hoehler $e t a l^{\text {in }}$ & & 1 & & & 4 & & & 5 & & 5 & & 3 & 4 & & 3 & 5 & 5 & 35 & Acute plus chronic low back pain & Positive \\
\hline Sims-Williams et al $l^{9}$ & 1 & & & & 4 & & 5 & 5 & & & 5 & & 8 & 2 & 5 & & & 35 & Acute plus chronic low back pain & Positive \\
\hline Zylbergold, Piper" & & 2 & & 3 & 4 & & & 5 & & & & & 6 & & 3 & 5 & 5 & 33 & Not mentioned & Negative \\
\hline Postacchini et al & & 2 & & & 2 & & & 5 & 5 & 5 & 5 & & 4 & & 5 & & & 33 & Acute plus chronic low back pain & $\begin{array}{l}\text { Positive (only in patients with acute back pain } \\
\text { not those with chronic pain) }\end{array}$ \\
\hline Rasmussen & 1 & 1 & & & 4 & & & 5 & 5 & & & & 4 & & 3 & 5 & 5 & 33 & Acute low back pain & Positive \\
\hline Farrell, Twomey; & 2 & 4 & & & 2 & & & 5 & 5 & & & & 6. & & 3 & & 5 & 32 & Acute low back pain & Positive \\
\hline Nwuga $^{\text {it }}$ & 2 & 3 & & & & & 10 & 5 & 5 & & & & 2 & 2 & 3 & & & 32 & $\begin{array}{l}\text { Acute low back pain (prolapsed } \\
\text { disc) }\end{array}$ & Positive \\
\hline Waterworth, Hunter" & 2 & 3 & & 3 & 4 & & & 5 & 5 & & & & 6 & & 3 & & & 31 & Acute low back pain & Negative \\
\hline Arkuszewski ${ }^{3 t}$ & & 1 & 2 & & 4 & & & 5 & & & & & 4 & & 5 & 5 & 5 & 31 & Acute plus chronic low back pain & Positive \\
\hline Buerger ${ }^{3-}$ & 1 & & & & & & 10 & 5 & 5 & & 5 & & 2 & & 3 & & & 31 & Not mentioned & Positive \\
\hline Tobis, Hoehler ${ }^{\text {ix }}$ & & & & & & & 5 & 5 & 5 & & 5 & 3 & 4 & & 3 & & & 30 & Not mentioned & Positive \\
\hline Bronfort ${ }^{i 4}$ & 1 & 2 & & & 2 & & & 5 & & & 5 & 3 & 2 & & 5 & & 5 & 30 & Acute plus chronic low back pain & No conclusion \\
\hline Kinalski et $a l^{\text {t }}$ & & & & & 4 & 6 & & & 5 & & & & 4 & & & & 5 & 24 & Not mentioned & Positive \\
\hline Godfrey et $a l^{+1}$ & 1 & 1 & & & 2 & & & 5 & & & & & 8 & 2 & 3 & & & 22 & Acute low back pain & Negative \\
\hline Siehl et a $t^{H^{2}}$ & 1 & 1 & & & & & & 5 & 5 & & 5 & & & & 5 & & & 22 & Not mentioned & $\begin{array}{l}\text { Positive (only in patients with no nerve root } \\
\text { compression not those with compression: }\end{array}$ \\
\hline $\begin{array}{l}\text { Rupert } e t \text { al } l^{43} \\
\text { Neck pain trials: }\end{array}$ & 1 & 1 & & & & & & & & 5 & 5 & 3 & 2 & & 3 & & & 20 & Acute plus chronic low back pain & Positive \\
\hline Sloop et al ${ }^{+4}$ & 2 & 1 & 2 & & 4 & & 5 & & & 5 & 5 & 5 & 4 & 4 & 3 & 5 & 5 & 50 & Chronic neck pain & Negative \\
\hline Nordemar, Thörner & 1 & 3 & & 3 & 4 & & 5 & 5 & 5 & & & & 4 & & 3 & 5 & 5 & 43 & Acute neck pain & Negative \\
\hline Brodin $^{\text {th }}$ & & 1 & 4 & & 2 & & 5 & 5 & 5 & & 5 & & 4 & & 3 & & 5 & 39 & Acute plus chronic neck pain & Positive \\
\hline Howe, Newcombe & & 3 & 2 & & & & 5 & 5 & & & & & 4 & 2 & 3 & & 5 & 29 & Acute plus chronic neck pain & Positive \\
\hline Mealy $e t a l^{+\times}$ & 1 & 2 & 4 & & 2 & & & 5 & & & & & 4 & & 3 & & 5 & 26 & Acute neck pain (whiplash) & Positive \\
\hline
\end{tabular}

*The labels acute and chronic are according to the authors of study. Classification might vary between the studies.

tConclusion of the author $(\mathrm{s})$ of the study. Positive conclusion=manipulation better than the control treatment; negative conclusion=manipulation worse than or equally effective as control treatment. 
treatments were mainly physiotherapeutic interventions (shortwave diathermy, massage, exercises) and drugs (analgesics). Thirty one trials compared manipulation with other conservative treatments. Of these, $15(48 \%)$ reported positive results in patients with both acute and chronic back and neck pain.

Of the three studies with method scores of $50-60$ points one reported negative results, and two studies reported positive results in only a subgroup. Among the eight studies with method scores of 40-50 points there were five reported negative results, two positive, and one positive results in only a subgroup.

Table IV presents the eight trials comparing manipulation with placebo. In most studies the placebo

TABLE III -Details of trials comparing manipulation with other conservative treatments

\begin{tabular}{|c|c|c|c|c|}
\hline Study & $\begin{array}{l}\text { Manipulation } \\
\text { (No of patients) }\end{array}$ & $\begin{array}{l}\text { Comparison treatment } \\
\text { (No of patients) }\end{array}$ & $\begin{array}{l}\text { Methods } \\
\text { score }\end{array}$ & Results` \\
\hline \multicolumn{5}{|l|}{ Back pain: } \\
\hline MacDonald, Bell' & (i) Osteopathic (49) & (ii) Exercises and advice on posture (46) & 56 & $\begin{array}{l}\text { All patients: no significant different recovery rates between treatment } \\
\text { groups. In subgroup with current attack duration of } 2-4 \text { weeks: } \% \text { of } \\
\text { patients recovered after one week; (i) } 46 \% \text {; (ii) } 17 \% \text {. }\end{array}$ \\
\hline Hadler $e$ e $a l^{\text {ss }}$ & (i) Rotational (26) & (ii) Spinal mobilisation (28) & 53 & $\begin{array}{l}\text { All patients: similar response rates according to Roland Morris } \\
\text { questionnaire. In subgroup with current attack duration of 2-4 weeks: } \\
\text { better results in manipulation group (i) after one week. }\end{array}$ \\
\hline $\begin{array}{l}\text { Bergquist-Ullman, } \\
\text { Larsson": }\end{array}$ & (i) Cyriax, Kaltenborn, Lewitt, Janda (72) & (ii) Back school (70) & 49 & Mean No of days until recovery: (i) $15 \cdot 8$; (ii) $14 \cdot 8$. \\
\hline Meade $e t a l^{1 /}$ & (i) Chiropractic (384) & (ii) Physiotherapy (357) $\dagger$ & 48 & $\begin{array}{l}\text { Difference in change in Oswestry questionnaire (mean score group (ii) } \\
\text { minus mean score group }(\mathrm{i}) \text { after } 6 \text { weeks, } 6 \text { months, } 1 \text { year, and } 2 \text { years: } \\
1 \cdot 69,3 \cdot 31,2 \cdot 09,7 \cdot 16 \text {, respectively. Manipulation significantly better } \\
\text { after } 6 \text { months and } 2 \text { years. }\end{array}$ \\
\hline Gibson et al ${ }^{14}$ & (i) Osteopathic (41) & (ii) Short wave diathermy (34) & 46 & $\%$ Of patients free of pain after 4 and 12 weeks: (i) $28 \%, 42 \%$; (ii) $28 \%, 37 \%$. \\
\hline Helliwell, Cunliffe"i & (i) Cyriax (6) & (ii) Analgesics (8) & 43 & $\begin{array}{l}\text { Combined symptom score (SD) }(\text { maximal severity }=28) \text { after } 1 \text { and } 4 \text { weeks: } \\
\text { (i) } 2 \cdot 6(2 \cdot 6), 3 \cdot 8(3 \cdot 3) ;(\text { ii) } 6 \cdot 17(7 \cdot 2), 2 \cdot 2(2 \cdot 5) \text {. No significant differences. }\end{array}$ \\
\hline Doran, Newell": & (i) At discretion of manipulator (116) & $\begin{array}{l}\text { (ii) Physiotherapy (114), (iii) analgesics } \\
\text { (113), (iv) corset (109) }\end{array}$ & 42 & $\begin{array}{l}\% \text { Of patients better after } 6 \text { and } 12 \text { weeks: (i) } 65 \%, 74 \% \text {; (ii) } 67 \%, 65 \% \text {; } \\
\text { (iii) } 58 \%, 76 \% \text {; (iv) } 77 \%, 83 \% \text {. }\end{array}$ \\
\hline Mathews $e t a l^{2}$ & (i) Cyriax (165) & (ii) Infrared heat (126) & 41 & $\begin{array}{l}\text { \% Of patients recovered after } 2 \text { weeks: In subgroup }(\mathrm{n}=58 \text { ) without } \\
\text { limitation in straight leg raising: (i) } 62 \% \text {; (ii) } 70 \% \text {. In subgroup }(\mathrm{n}=233 \text { ) } \\
\text { with limitation in straight leg raising: (i) } 80 \% \text {; (ii) } 67 \% \text {. Manipulation } \\
\text { gave significant beneficial effect in subgroup with limited straight leg } \\
\text { raising. }\end{array}$ \\
\hline Evans $e t a l^{4}$ & (i) Rotational (15) & (ii) Analgesics (17) & 40 & $\begin{array}{l}\text { No of patients assessing treatment as effective after } 3 \text { weeks: (i) } 9 \text {; (ii) } 3 \text {. } \\
\text { Significant. }\end{array}$ \\
\hline Coxhead et al $l^{\text {h }}$ & (i) Maitland (155) & (ii) Exercises or corset or traction (137) & 38 & $\begin{array}{l}\text { No of patients reporting to feel better compared with baseline after } 4 \text { weeks } \\
\text { and } 4 \text { months: (i) } 127,100 \text {; (ii) } 100,81 \text {. No significant difference. }\end{array}$ \\
\hline Waagen et al ${ }^{2}$ & (i) Chiropractic (9) & $\begin{array}{l}\text { (ii) Massage and sham manipulation } \\
\text { (10) }\end{array}$ & 37 & $\begin{array}{l}\text { Improvement on visual analogue scale }(10 \mathrm{~cm}) \text { after } 2 \text { weeks: (i) } 2 \cdot 3 \text {; (ii) } 0.6 \text {. } \\
\text { Manipulation significantly better. }\end{array}$ \\
\hline Hochler et $a l^{2 \kappa}$ & (i) Rotational (56) & (ii) Massage (39) & 35 & $\begin{array}{l}\text { No of patients reporting moderate to severe pain at discharge (variable) and } \\
3 \text { weeks after discharge: (i) } 17,21 \text {; (ii) } 29,48 \text {. Manipulation significantly } \\
\text { better after first treatment. No differences at discharge and } 3 \text { weeks later. }\end{array}$ \\
\hline Zylbergold, Piper ${ }^{30}$ & (i) Rotational (8) & (ii) Heat and exercises (10) & 33 & $\begin{array}{l}\text { Improvement (SD) on } 5 \text { point pain scale: }(\mathrm{i})-1 \cdot 5(0 \cdot 1) ;(\mathrm{ii})-1 \cdot 0(0 \cdot 85) \text {. No } \\
\text { significant differences but results indicate slight advantage for } \\
\text { manipulation. }\end{array}$ \\
\hline Postacchini $e t a l^{31}$ & (i) Chiropractic (87) & $\begin{array}{l}\text { (ii) Physiotherapy ( } 78) \text {, (iii) drugs }(81) \text {, } \\
\text { (iv) bedrest }(29),(\mathrm{v}) \text { back school }(50)\end{array}$ & 33 & $\begin{array}{l}\text { Mean improvement on combined pain, disability, and spinal mobility score } \\
\text { (5-32) after } 3 \text { weeks, } 2 \text { months, and } 6 \text { months. In subgroup with acute } \\
\text { pain: (i) } 7 \cdot 5,9 \cdot 7,12 \cdot 3 ; \text { (ii) } 5 \cdot 0,8 \cdot 4,10 \cdot 2 ; \text { (iii) } 3 \cdot 0,10 \cdot 7,14 \cdot 0 ; \text { (iv) } 5 \cdot 4,7 \cdot 5 \text {, } \\
7 \cdot 3 \text {. Manipulation significantly better (short term) only in subgroup with } \\
\text { acute pain only after } 3 \text { weeks. In subgroup with chronic pain: (i) } 2 \cdot 2,2 \cdot 6 \text {, } \\
4 \cdot 3 ; \text { (ii) } 3 \cdot 9,4 \cdot 2,6 \cdot 0 ; \text { (iii) } 2 \cdot 6,2 \cdot 2,4 \cdot 0 ; \text { (v) } 0 \cdot 5,4 \cdot 6,8 \cdot 9 \text {. Manipulation not } \\
\text { better in subgroup with chronic pain. }\end{array}$ \\
\hline Rasmussen $^{32}$ & (i) Rotational (12) & (ii) Short wave diathermy (12) & 33 & $\begin{array}{l}\text { No (\%) of patients totally restored after } 14 \text { days: (i) } 11(92 \%) \text {; (ii) } 3(25 \%) \text {. } \\
\text { Manipulation significantly superior. }\end{array}$ \\
\hline Farrell, Twomey ${ }^{33}$ & (i) Stoddard, Maitland (24) & $\begin{array}{l}\text { (ii) Short wave diathermy and exercises } \\
\text { (24) }\end{array}$ & 32 & $\begin{array}{l}\text { Manipulation group was symptom free in significantly less days. (Data in } \\
\text { graphs). }\end{array}$ \\
\hline Nwuga't & (i) Rotational (26) & $\begin{array}{l}\text { (ii) Short wave diathermy and exercises } \\
\text { (25) }\end{array}$ & 32 & $\begin{array}{l}\text { Improvement in spinal flexion and straight leg raising after } 6 \text { weeks: } \\
\text { (i) } 34^{\circ}, 39^{\circ} \text {; (ii) } 13^{\circ}, 4^{\circ} \text {. Manipulation significantly better than comparison } \\
\text { treatment. }\end{array}$ \\
\hline Waterworth, Hunter ${ }^{35}$ & (i) At discretion of physiotherapist (38) & $\begin{array}{l}\text { (ii) Short wave diathermy, ultrasound, } \\
\text { and exercise (34) (iii) Non-steroidal } \\
\text { antiinflammatory drugs ( } 36 \text { ) }\end{array}$ & 31 & $\begin{array}{l}\text { Mean change in pain intensity on } 4 \text { point scale after } 4 \text { and } 12 \text { days: (i) }-1 \cdot 1 \text {, } \\
-1 \cdot 7 ; \text { (ii) }-0 \cdot 9,-1 \cdot 6 ; \text { (iii) }-0 \cdot 9,-1 \cdot 7 \text {. No significant differences in pain } \\
\text { and mobility. }\end{array}$ \\
\hline Arkuszewski ${ }^{i n}$ & (i) Lewit (50) & $\begin{array}{l}\text { (ii) Bedrest, analgesics, and massage } \\
\text { (50) }\end{array}$ & 31 & $\begin{array}{l}\text { Pain severity (SD) on } 4 \text { point scale }(0-3) \text { immediately after treatment and } \\
\text { after } 6 \text { months; (i) } 0 \cdot 6(0 \cdot 5), 0 \cdot 7(0 \cdot 6) ; \text { (ii) } 1 \cdot 0(0 \cdot 4), 1 \cdot 0(0 \cdot 5) \text {. Improvement } \\
\text { was significantly greater in manipulation group. }\end{array}$ \\
\hline Buerger ${ }^{2}$ & (i) Rotational (-) & (ii) Massage and sham treatment $(-)$ & 31 & $\begin{array}{l}\% \text { Of patients who feel better after last treatment and } 5 \text { days later: (i) } 83 \% \text {, } \\
52 \% \text {; (ii) } 67 \%, 66 \% \text {. Manipulation significantly superior only } \\
\text { immediately after last treatment. }\end{array}$ \\
\hline Tobis, Hochler ${ }^{38}$ & (i) Rotational (-) & (ii) Massage (-) & 30 & $\begin{array}{l}\text { Manipulation significantly better result in pain relief immediately after } \\
\text { treatment. After } 5 \text { days no significant differences (data in graphs). }\end{array}$ \\
\hline Bronfort ${ }^{19}$ & (i) Chiropractic (10) & $\begin{array}{l}\text { (ii) Medical treatment (for example, } \\
\text { drugs) (9) }\end{array}$ & 30 & $\begin{array}{l}\% \text { Of patients improved after } 1,3 \text {, and } 6 \text { months; (i) } 70 \%, 70 \%, 80 \% \text {; } \\
\text { (ii) } 55 \% \text {; } 66 \%, 66 \% \text {. Author gives no conclusion because of small sample } \\
\text { size. }\end{array}$ \\
\hline Kinalski $e t a l^{+i}$ & (i) Janda, Lewitt (61) & (ii) Physiotherapy (50) & 24 & $\begin{array}{l}\% \text { Of patients with good result (low pain grade) after treatment: (i) } 84 \% \text {, } \\
\text { (ii) } 78 \% \text {. }\end{array}$ \\
\hline Godfrey $e t a l^{4 !}$ & (i) Rotational manipulation (44) & $\begin{array}{l}\text { (ii) Massage and electrical stimulation } \\
\text { (37) }\end{array}$ & 22 & $\begin{array}{l}\% \text { Of patients with moderate or marked improvement in general symptoms } \\
\text { on a } 5 \text { point scale after } 2 \text { weeks: (i) } 77 \% \text {; (ii) } 70 \% \text {. Five other indexes also } \\
\text { showed no significant differences. }\end{array}$ \\
\hline Sichl et alt? & (i) Osteopathic (21) & $\begin{array}{l}\text { (ii) Conservative treatment (7), (iii) } \\
\text { Surgery (19) }\end{array}$ & 22 & $\begin{array}{l}\% \text { Of patients showing electromyographic and clinical improvement after } 6 \\
\text { and } 12 \text { months; (i) } 14 \% \text {; (ii) } 0 \% \text {; (iii) } 47 \% \text {. }\end{array}$ \\
\hline Rupert et $a l^{s:}$ & (i) Chiropractic (-) & $\begin{array}{l}\text { (ii) Drugs and bed rest }(-) \text {, (iii) Home } \\
\text { care instructions }(-)\end{array}$ & 20 & $\begin{array}{l}\text { Improvement on pain visual analogue scale during treatment: group (i) } \\
\text { more pain reduction than groups (ii) and (iii). Data in graphs. }\end{array}$ \\
\hline $\begin{array}{l}\text { Neck pain: } \\
\quad \text { Sloop at al }\end{array}$ & (i) Cyriax, Maigne, Maitland (21) & (ii) Diazepam (18) & 50 & $\begin{array}{l}\% \text { Of patients reporting that the treatment helped after } 3 \text { weeks; (i) } 57 \% \text {; } \\
\text { (ii) } 28 \% \text {. No significant difference. }\end{array}$ \\
\hline Nordemar, Thörner & (i) Mobilisation therapy (10) & $\begin{array}{l}\text { (ii) Transcutaneous nerve stimulation } \\
\text { (10), (iii) neck collar }(10)\end{array}$ & 43 & $\begin{array}{l}\text { Mean (SD) on pain visual analogue scale after } 1 \text { and } 6 \text { weeks: (i) } 18(25), 0 \text {; } \\
\text { (ii) } 17(19), 0 \text {; (iii) } 34(45), 0 \text {. No significant difference. }\end{array}$ \\
\hline Brodin ${ }^{\text {th }}$ & (i) Stoddard (23) & $\begin{array}{l}\text { (ii) analgesics }(23) \text {, (iii) analgesics and } \\
\text { mock manual therapy (17) }\end{array}$ & 39 & $\begin{array}{l}\% \text { Of patients reporting no pain one week after final treatment: (i) } 48 \% \text {; } \\
\text { (ii) } 22 \% \text {; (iii) } 12 \% \text {. Manipulation significantly better. }\end{array}$ \\
\hline Howe, Newcombe ${ }^{+}$ & (i) Bourdillon (26) & (ii) Analgesics (26) & 29 & $\begin{array}{l}\% \text { Of patients improved (pain measurement) immediately and } 1 \text { and } 3 \text { weeks } \\
\text { after treatment: (i) } 68 \%, 74 \%, 76 \% ; \text { (ii) } 6 \%, 60 \%, 58 \% \text {. Manipulation } \\
\text { significantly better only immediately after treatment. }\end{array}$ \\
\hline Mealy et al $l^{* *}$ & (i) Maitland (31) & (ii) Rest and cervical collar (30) & 26 & $\begin{array}{l}\text { Mean (SE) pain visual analogue scale } 4 \text { and } 8 \text { weeks after treatment: } \\
\text { (i) } 2 \cdot 85(0 \cdot 57), 1 \cdot 69(0 \cdot 43) ; \text { (ii) } 5 \cdot 08(0 \cdot 48), 3 \cdot 94(0 \cdot 58) \text {. Manipulation } \\
\text { significantly better. }\end{array}$ \\
\hline
\end{tabular}

${ }^{\star}$ Results of the most important outcome measure according to the author(s) of the study. When not explicitly stated presentation of pain or a global measure of improvement. Significant mean $\mathrm{p}<0 \cdot 05$ 


\begin{tabular}{|c|c|c|c|c|}
\hline Study & $\begin{array}{l}\text { Manipulation } \\
\text { (No of patients) }\end{array}$ & $\begin{array}{c}\text { Placebo } \\
\text { (No of patients) }\end{array}$ & $\begin{array}{l}\text { Methods } \\
\text { score }\end{array}$ & Results \\
\hline Ongley $e t a l^{\text {th }}$ & (i) Bourdillon (40) & (ii) Non-forceful manipulation (41) & 50 & $\begin{array}{l}\text { Mean (SE) pain on visual analogue scale after } 1,3 \text {, and } 6 \text { months: (i) } 2 \cdot 1(0 \cdot 2) \text {, } \\
1 \cdot 8(0 \cdot 2), 1 \cdot 5(0 \cdot 2) ; \text { (ii) } 3 \cdot 1(0 \cdot 3), 2 \cdot 9(0 \cdot 3), 3 \cdot 1(0 \cdot 3) \text {. All differences significant }\end{array}$ \\
\hline Bergquist, Larsson ${ }^{17}$ & $\begin{array}{l}\text { (i) Cyriax, Kaltenborn, Lewitt, } \\
\text { Janda (72) }\end{array}$ & $\begin{array}{l}\text { (ii) Short wave diathermy at lowest } \\
\text { intensity (75) }\end{array}$ & 49 & Mean No of days until recovery: (i) $15 \cdot 8$; (ii) $28 \cdot 7$. Difference significant. \\
\hline Gibson et al ${ }^{19}$ & (i) Osteopathic (41) & $\begin{array}{l}\text { (ii) Detuned short wave diathermy } \\
\text { (34) }\end{array}$ & 46 & $\%$ Of patients free of pain after 4 and 12 weeks: (i) $28 \%, 42 \%$; (ii) $27 \%, 44 \%$. \\
\hline Sims-Williams et $a l^{20}$ & (i) Maitland (48) & (ii) Microwave at lowest setting (46) & 45 & $\begin{array}{l}\text { No of patients improved after } 4 \text { and } 12 \text { weeks: (i) } 29,28 \text {; (ii) } 25,27 \text {. Not significantly } \\
\text { different. Also no evidence that manipulation produced any long term (one year) } \\
\text { benefit. }\end{array}$ \\
\hline Glover $e$ a $a l^{2 s}$ & (i) Rotational (43) & $\begin{array}{l}\text { (ii) Detuned short wave diathermy } \\
\text { (41) }\end{array}$ & 39 & $\begin{array}{l}\text { Mean pain relief (\%) on visual analogue scale immediately after treatment and after } 3 \\
\text { and } 7 \text { days: (i) } 34 \%, 50 \%, 75 \% \text {; (ii) } 22 \%, 56 \%, 80 \% \text {. Apart from slight immediate } \\
\text { improvement after treatment no benefit from manipulation. }\end{array}$ \\
\hline Sims-Williams et al $^{99}$ & (i) Maitland (47) & (ii) Microwave at lowest setting (47) & 35 & $\begin{array}{l}\text { No of patients improved after } 4 \text { and } 12 \text { weeks: (i) } 39,26 \text {; (ii) } 32,22 \text {. After } 4 \text { weeks the } \\
\text { differences were of borderline significance. After } 12 \text { weeks the differences } \\
\text { disappeared. }\end{array}$ \\
\hline Postacchini $e t a l^{31}$ & (i) Chiropractic (87) & (ii) Antioedema gel (73) & 33 & $\begin{array}{l}\text { Mean improvement on combined pain, disability, spinal mobility score (5-35) after } 3 \\
\text { weeks, } 2 \text { months, and } 6 \text { months. In subgroup with acute pain: (i) } 7 \cdot 5,9 \cdot 7,12 \cdot 3 ; \text { (ii) } \\
1 \cdot 8,7 \cdot 3,11 \cdot 0 \text {. In subgroup with chronic pain: (i) } 2 \cdot 2,2 \cdot 6,4 \cdot 3 ; \text { (ii) } 0 \cdot 7,1 \cdot 2,2 \cdot 0 \text {. } \\
\text { Manipulation was significantly better only in subgroup with acute pain after } 3 \\
\text { weeks. }\end{array}$ \\
\hline Rupert $e t a l^{43}$ & (i) Chiropractic (-) & (ii) Sham manipulation, massage (-) & 20 & $\begin{array}{l}\text { Improvement on pain visual analogue scale during treatment: more pain reduction in } \\
\text { group receiving manipulation (data in graphs). }\end{array}$ \\
\hline
\end{tabular}

${ }^{\star}$ Results of the most important outcome measure according to the author(s) of the study. When not explicitly stated presentation of pain or a global measure of improvement. Significant mean $\mathrm{p}<0 \cdot 05$.

consisted of detuned shortwave diathermy. Four $(50 \%)$ studies reported positive results, one positive results in only a subgroup, and three negative results. The study populations included patients with both acute and chronic conditions. Sims-Williams et al in two studies with comparable designs, report positive results of manipulation (compared with microwave at lowest setting) for patients in general practice but not for hospital patients. ${ }^{20}{ }^{29}$ Long term effects were found only in study by Ongley et al..$^{10}$ In their study, however, manipulation was only one part of a treatment regimen which also included injections and exercises and the effect of manipulation could not be isolated.

\section{Discussion}

The value of a review of the literature depends on the success in obtaining the results of all studies that have been conducted on the subject at issue. Such studies are subject to bias caused by the outcomes of published and unpublished studies differing (publication bias). There are indications that, especially, small clinical trials with positive results are more likely to be published. ${ }^{49}$ Although we put much effort into obtaining all the available published randomised clinical trials, we may have missed both published and unpublished trials, the results of which might differ from those of the trials we have presented. No register of trials that are or will be carried out exists in this subject. Thus it is unknown whether there are trials

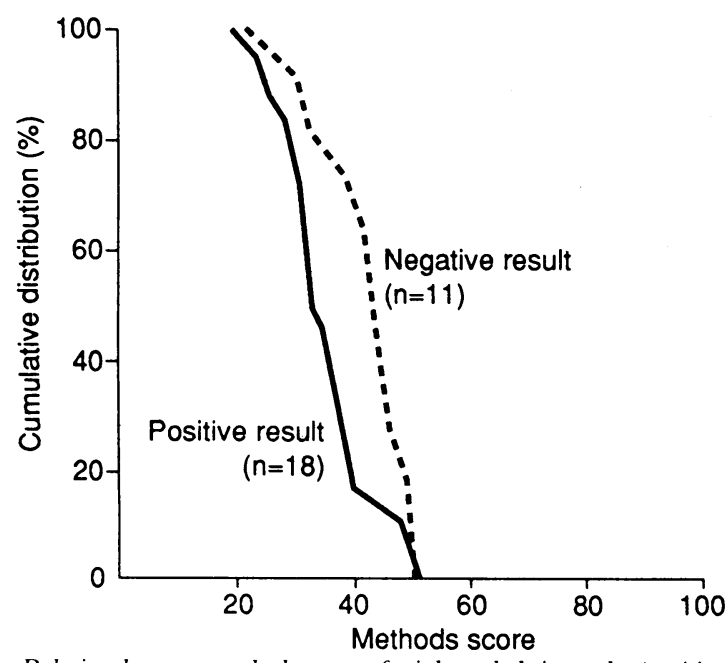

Relation between methods score of trials and their results (positive results shows manipulation is better than reference treatment, negative result shows manipulation is no better or worse than reference treatment) that have not been published because of negative results. We did not find any completed unpublished studies, although our search was not primarily focused to trace them.

Although there were many randomised clinical trials of manipulation, most showed major methodological flaws. The criteria that we used to assess methods are based on the requirements for high quality of intervention research in this subject. Although the standard of 100 points is probably difficult to reach in this area of research, it was disappointing to find that most trials scored less than 50 points. In future studies more attention should be given to the description of drop outs, the size of the study population, the use of placebo groups, and blinded measurements of effect. It seems difficult to develop a placebo manipulation treatment that has no specific effect and is trusted by the patients. Some authors supplied high doses of diazepam to patients, thus making the patients amnesic for the procedure and unaware of the treatment given. ${ }^{1644}$ Other authors include non-therapeutic massage ${ }^{43}$ or massage in combination with sham adjustments $^{27}$ to simulate the effect of laying on of hands These efforts can be criticised on the grounds that they might have some therapeutic effects. Detuned short wave diathermy seems to be a placebo therapy which patients can trust and which has no specific effects, but this has little similarity to manipulation.

\section{ANALYSIS OF TRIALS}

The other published reviews of the efficacy of manipulation have summarised only seven to 17 of the 35 randomised clinical trials we included in our analysis. Greenland et al,${ }^{50}$ Brunarski, ${ }^{51}$ and Di Fabio ${ }^{53}$ stated that adequate randomised clinical trials are still needed for a valid assessment of the efficacy of manipulation. Ottenbacher and Di Fabio conducted a quantitative meta-analysis in which the results from nine trials were pooled statistically and concluded that there is only limited empirical support for the efficacy of manipulation. ${ }^{6}$ We chose not to pool statistically the results of the available trials, mainly because we do not like the idea of pooling data from studies of high and low methodological quality. This decision was supported by our finding that the trials which reported positive results of manipulation more often had relatively low method scores. We did not pool the results of the subgroup of trials with a relatively high method score as we did not think that the patient characteristics and treatments used in these trials showed enough similarity to permit pooling of their data.

The methods used in our review are similar to those we used in a review on physiotherapy exercises. ${ }^{53}$ The 
only difference is that in this review on manipulation we gave five points if the article indicated that the manipulative treatment had been carried out by a qualified or experienced manipulative therapist, or both. These five points were withdrawn from the 17 points that could be earned for the size of the study population, which left 12 points for this criterion. The weighting attached to the criteria remains arbitrarily chosen. For example, description of an adequate randomisation procedure was given low weight (four points) because all the studies included were randomised clinical trials and thus satisfied our demand of random allocation of the participants. Studies could, however, earn points with a proper description of an adequate randomisation procedure. The assessment of trials by blinded reviewers was not difficult, and after a consensus meeting the inter-observer agreement was almost perfect.

Most trials reported only short term effects. The studies that do include a long term follow up mostly show no positive results. Many studies, especially the ones with relatively higher methods scores $(>40$ points) report negative results, even when compared with placebo. The negative results might be caused partly by relatively small study populations, which make it difficult to detect treatment differences between manipulation and reference treatments. ${ }^{54}$ Thus the information in the figure must be interpreted with caution as the labelling of studies as negative might be influenced by the lower power of small trials. The results of all the trials presented indicate that manipulation is not consistently better than other therapies. Manipulation may be effective in only certain subgroups of patients with back and neck pain. The findings of Sims-Williams et al seem to support this suggestion. ${ }^{2029}$ Furthermore, five trials reported better results for manipulation in only subgroups. It is still unclear, however, which subgroup(s) benefit most from manipulation. Positive and negative results are reported for patients with both acute and chronic back and neck pain. In two studies with relatively high method scores favourable results of manipulation were found for a subgroup of patients with low back pain of two to four weeks' duration. ${ }^{14}{ }^{15}$ Although the findings from subgroup analyses should be interpreted cautiously,${ }^{58}$ this seems to be an interesting finding for further research.

In the meantime we conclude that, although there are some promising results, so far the efficacy of manipulation has not been convincingly shown. Any further research should pay more attention to the methodological quality of the study design.

\section{Appendix}

Details of criteria listed in table I. Each criterion must be applied independently of the other criteria.

A Description of inclusion and exclusion criteria (1 point). Restriction to a homogeneous study population (1 point).

B Comparability for duration of complaints, value of outcome measures, age, recurrences, and radiating complaints ( 1 point each).

C Randomisation procedure described ( 2 points). Randomisation procedure which excludes bias-for example, sealed envelopes - ( 2 points).

D Information about which group patients withdrew from and reason for withdrawal.

E Loss to follow up: all randomised patients minus the number of patients at main point of measurement of the main outcome measure, divided by all randomised patients, multiplied by 100 .

F Smallest group immediately after randomisation.

G Manipulative treatment explicitly described (5 points). All reference treatments explicitly described (5 points).

$\mathrm{H}$ Comparison with an established treatment.

I Other physical treatments or medical interventions are avoided in the design of the study (except analgesics; advice on posture; or use at home of heat, rest, or a routine exercise scheme).

J Comparison with placebo.

K Mentioning of qualified education or experience of the manipulative therapist(s), or both.

L Placebo controlled study: attempt at blinding ( 3 points), blinding evaluated and fully successful ( 2 points).

Pragmatic study: patients fully naive ( 3 points) or time restriction (no manipulative treatment for at least one year) ( 2 points); naiveness evaluated and fully successful ( 2 points).

M Use (measured and reported) of pain, global measurement of improvement, functional status (activities of daily living), spinal mobility, use of drugs and medical services ( 2 points each).

$\mathrm{N}$ Each blinded measurement mentioned under point $M$ earns 2 points.

O Outcome of measures assessed during or just after treatment (3 points). Outcome of measures assessed six months or longer ( 2 points)

P When loss to follow up is less than 10\%: analyses on all randomised patients for main outcome measures, and on the most important points of measurement minus missing values, irrespective of non-compliance and cointerventions. When loss to follow up is greater than $10 \%$ : intention to treat as well as an alternative analysis that accounts for missing values.

$Q$ For main outcome measures, and at main times of measurement. In the case of (semi)continuous variables; presentation of the mean or median with standard error or centiles.

1 Kelsey JL, White AA. Epidemiology and impact of low-back pain Spine 1980;5:133-42.

2 Waddell G. A new clinical model for the treatment of low-back pain Spine 1987;12:632-44.

3 Frymoyer JW. Back pain and sciatica. N Engl F Med 1988;318:291-300.

4 Deyo RA. Conservative therapy for low back pain. FAMA 1983;250: 1057-62.

5 Spitzer WO, Leblanc FE, Dupuis M, eds. Scientific approach to the assessment and management of activity-related spinal disorders. Spine 1987;7(suppl): 1-59.

6 Ottenbacher K, Di Fabio RP. Efficacy of spinal manipulation/mobilization therapy: a meta-analysis. Spine 1985;10:833-7.

7 Jayson MIV, Sims-Williams H, Young S, Baddeley H, Collins F Mobilization and manipulation for low-back pain. Spine 1981;6: Mo9-16.

8 Gatterman MI. Chiropractic management of spine related disorders. Baltimore: Williams and Wilkins, 1990:37-5

9 Meinert CL. Clinical trials: design, conduct and analysis. New York: Oxford University Press, 1986.

10 Feinstein AR. Clinical epidemiology: the architecture of clinical research. Philadelphia: W B Saunders, 1985.

11 Ter Riet G, Kleijnen J, Knipschild P. Acupuncture and chronic pain: criteria-based meta-analysis. $\mathcal{F}$ Clin Epidemiol 1990;43:1191-9.

12 Mathews W, Morkel M, Mathews J. Manipulation and traction for lumbago and sciatica: physiotherapeutic techniques used in two controlled trials. Physiotherapy Practice 1988;4:201-6.

13 Brodin H. Cervical pain and mobilization. Int $\mathcal{F}$ Rehab Res 1984;7: 190-1.

14 MacDonald RS, Bell CMJ. An open controlled assessment of osteopathic manipulation in nonspecific low-back pain. Spine 1990;15: 364-70.

15 Hadler NM, Curtis P, Gillings DB, Stinnett S. A benefit of spinal manipulation as adjunctive therapy for acute low-back pain: a stratified controlled trial. Spine 1987;12:703-5.

16 Ongley MJ, Klein RG, Dorman TA, Eck BC, Hubert LJ. A new O approach to the treatment of chronic low back pain. Lancet 1987; ii: 143-6.

17 Bergquist-Ullman M, Larsson U. Acute low back pain in industry: a controlled prospective study with special reference to therapy and $N$ confounding factors. Acta Orthop Scand 1977;170(suppl):11-117.

18 Meade TW, Dyer S, Browne W, Townsend J, Frank AO. Low back pain of mechanical origin: randomised comparison of chiropractic and $O$ hospital outpatient treatment. BMF 1990;300:1431-7.

19 Gibson T, Grahame R, Harkness J, Woo P, Blagrave P, Hills R. Controlled comparison of short-wave diathermy treatment with $\mathbb{D}$ osteopathic treatment in non-specific low back pain. Lancet 1985; : $1258-61$.

20 Sims-Williams H, Jayson MIV, Young SMS, Baddeley H, Collins E. Controlled trial of mobilisation and manipulation for low back pain: hospital patients. BMF 1979;ii: 1318-20.

21 Helliwell PS, Cunliffe G. Manipulation in low back pain. Physician 1987; A pril: $187-8$

22 Doran DML, Newell DJ. Manipulation in treatment of low back pain: a multicentre study. BMF 1975;ii:161-4

23 Mathews JA, Mills SB, Jenkins VM, Grimes SM, Morkel MJ, Mathews W, et al. Back pain and sciatica: controlled trials of manipulation, traction, sclerosant and epidural injections. Br f Rheumatol 1987;26: 416-23.

24 Evans DP, Burke MS, Lloyd KN, Roberts EE, Roberts GM. Lumbar spinal manipulation on trial. 1: Clinical assessment. Rheumatol Rehab? 1978;17:46-53

25 Glover JR, Morris JG, Khosla T. Back pain: a randomized clinical tria of rotational manipulation of the trunk. Br F Ind Med 1974;31:59-64. 
26 Coxhead CE, Inskip H, Meade TW, North WRS, Troup JDG. Multicentre trial of physiotherapy in the management of sciatic symptoms. Lancet $1981 ;$ i: $1065-8$.

27 Waagen GN, Haldeman S, Cook G, Lopez D, DeBoer KF. Short term trial of chiropractic adjustments for the relief of chronic low back pain. Manual Medicine 1986;ii:63-7.

28 Hoehler GK, Tobis JS, Buerger AA. Spinal manipulation for low back pain. FAMA 1981;245:1835-8.

29 Sims-Williams H, Jayson MIV, Young SMS, Baddeley H, Collins E Controlled trial of mobilisation and manipulation for patients with low back pain in general practice. $B M$ I 1978 ;ii: $1338-40$.

30 Zylbergold RS, Piper MC. Lumbar disc disease: comparative analysis of physical therapy treatments. Arch Phys Med Rehabil 1981;62:176-9.

31 Postacchini F, Faccini M, Palieri P. Efficacy of various forms of

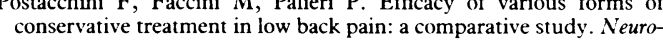
conservative treatment in
orthopedics 1988;6:28-35.

32 Rasmussen GG. Manipulation in treatment of low back pain: a randomized clinical trial. Manual Medicine 1979;i:8-10.

33 Farrell JP, Twomey LT. Acute low back pain: comparison of two conservative treatment approaches. Med f A ust 1982;1:160-4.

34 Nwuga VCB. Relative therapeutic efficacy of vertebral manipulation and conventional treatment in back pain management. Am $\mathcal{F}$ Physical Med 1982;1:160-4.

35 Waterworth RF, Hunter IA. An open study of diflunisal, conservative and manipulative therapy in the management of acute mechanical low back pain. $N Z$ Med $\mathcal{F} 1985 ; 98: 372-5$.

36 Arkuszewski $Z$. The efficacy of manual treatment in low back pain: a clinical trial. Manual Medicine 1986;ii:68-71.

37 Buerger AA. A controlled trial of rotational manipulation in low back pain. Manual Medicine 1980;ii:17-26.

38 Tobis JS, Hoehler FK. Musculoskeletal manipulation in the treatment of low back pain. Bull N Y Acad Med 1983;7:660-8.

39 Brontfort GDC. Chiropractic versus general medical treatment of low back pain: a small scale controlled clinical trial. American fournal of Chiropractic Medicine 1989;2:145-50.

40 Kinalski R, Kuwik W, Pietrzak D. The comparison of the results of manual therapy versus physiotherapy methods used in treatment of manual therapy versus physiotherapy methods used in treatment of
patients with low back pain syndromes. Manual Medicine 1989;iv: 44-6.
41 Godfrey CM, Morgan PP, Schatzker J. A randomized trial of manipulation for low-back pain in a medical setting. Spine 1984;9:301-4

42 Siehl D, Olson DR, Ross HE, Rockwood EE. Manipulation of the lumbar spine with the patient under general anesthesia: Evaluation by electromyography and clinical-neurologic examination of its use for lumbar nerve root compression syndrome. Fournal of the American Osteopath Association 1971;70:433-40.

43 Rupert RL, Wagnon R, Thompson P, Ezzeldin MT. Chiropractic adjustments: results of a controlled clinical trial in Egypt. Internation Review of Chiropractic 1985; Winter:58-60.

44 Sloop PR, Smith DS, Goldenberg E, Doré C. Manipulation for chronic neck pain: a double-blind controlled study. Spine 1982;7:532-5.

45 Nordemar R, Thörner C. Treatment of acute cervical pain: a comparative group study. Pain 1980;10:93-101.

46 Brodin H.Cervical pain and mobilization. Med Phys 1983:6:67-72

47 Howe $\mathrm{DH}$, Newcombe $\mathrm{R}$. Manipulation of the cervical spine $f R$ Co Gen Pract 1983;33:574-9.

48 Mealy $\mathrm{K}$, Brennan $\mathrm{H}$, Fenelon GCC Early mobilication of acu whiplash injuries. BMF 1986;292:656-7.

9 Dickersin $\mathrm{K}$. The existence of publication bias and risk factors for its occurrence. $f A M A$ 1990,263:1385-9.

50 Greenland S, Reisbord LS, Haldeman S, Buerger AA. Controlled clinical trials of manipulation: a review and a proposal. F Occup Med 1980;22:670-6

51 Brunarski DJ. Clinical trials of spinal manipulation: a critical appraisa and review of the literature. $\mathcal{F}$ Manipulative Physiol Ther 1984;7:243-9.

52 Di Fabio RP. Clinical assessment of manipulation and mobilization of the lumbar spine: a critical review of the literature. Phys Ther 1986;66:51-4.

53 Koes BW, Bouter LM, van der Heijden GJMG, Knipschild PG. Physiotherapy exercises and back pain: a blinded review. $B M Y$ 1991;30:1572-6.

54 Freiman JA, Chalmers TC, Smith H, Kuebler RR. The importance of beta, the type II error and sample size in the design and interpretation of the randomized controlled trial. N Engl F Med 1978;299:690-4.

55 Pocock SJ, Hughes MD, Lee RJ. Statistical problems in the reporting of clinical trials. N Engl ff Med 1987;317:426-32.

(Accepted 4 September 1991)

\section{Dynamics of spread of HIV-I infection in a rural district of Uganda}

Maria J Wawer, David Serwadda, Stanley D Musgrave, Joseph K Konde-Lule, Maako Musagara, Nelson K Sewankambo

Abstract

Objective-To define the geographical distribution of HIV infection and the community characteristics associated with HIV prevalence in a rural population of Uganda.

Design-Seroprevalence survey and interviews of the population aged 13 years and older in 21 randomly selected clusters.

Setting-Rural population of Rakai district, south west Uganda.

Subjects-1292 adults, of whom 594 men and 698 women gave a blood sample and answered the questionnaire.

Main outcome measures-HIV status determined by ELISA and western blotting in relation to community characteristics.

Results - The weighted seroprevalence of HIV for the district was $12.6 \%$ with prevalence by cluster varying from $1 \cdot 2 \%$ to $52 \cdot 8 \%$. Seroprevalence was highest in main road trading centres (men $26 \%$, women $47 \%$ ), intermediate in rural trading villages on secondary roads (men $22 \%$, women $29 \%$ ), and lowest in rural agricultural villages (men $8 \%$, women 9\%). For both men and women, multiple regression showed a strong negative association between cluster seroprevalence and the proportion of the population employed in agriculture $(\beta=-0.677$ for men, -0.807 for women). Among women, cluster seroprevalence increased with a higher proportion of the population reporting multiple sex partners $(\beta=0.814)$, external travel $(\beta=0.579)$, and injections $(\beta=0.483)$.

Conclusions-Community characteristics, particularly the proportion of the population in agriculture, are associated with HIV prevalence and can be used for targeting interventions. The seroprevalences of HIV suggest spread of infection from main road trading centres, through intermediate trading villages, to rural agricultural villages.

\section{Introduction}

Despite reports of high rates of HIV infection from clinical and urban settings in Africa ${ }^{1-6}$ data on rural populations are still scarce. Limited information suggests that HIV infection is spreading rapidly outside urban centres in some east and central African countries. ${ }^{78}$ As about $70 \%$ of the sub-Saharan African population does not live in cities, it is critical to understand the dynamics of the HIV epidemic in rural areas. Trading villages along main roads represent one obvious reservoir of infection outside the main urban areas; their importance as focuses of infection, related in part to commercial sex between local women and long distance truck drivers, has been reported in Uganda. ${ }^{910}$ However, the spread of HIV from these centres and the distribution of infection within agricultural areas have not been adequately defined. We attempted to define the spread of HIV in Rakai district, south western Uganda, where a random sample of residents have been enrolled in a longitudinal cohort study of HIV-I transmission and prevention.

\section{Subjects and methods}

SAMPLING

Rakai district has a population of 350000 , is about two hours by road from Kampala, and borders on northern Tanzania. Although Rakai is primarily rural, it is traversed by major roads that carry traffic from Tanzania, Rwanda, Kenya, and Lake Victoria (figure). The district contains 780 level one resistance committees, which are the smallest administrative units in 